\title{
Excess risk of hospital admission for cardiovascular disease within the first 7 years from onset of inflammatory polyarthritis
}

\author{
Jarrod Franklin, ${ }^{1}$ Tracey M Farragher, ${ }^{1}$ Mark Lunt, ${ }^{1}$ Elizabeth M Camacho, ${ }^{1}$ Diane \\ Bunn, ${ }^{1,2}$ Tarnya Marshall, ${ }^{2}$ Deborah P M Symmons ${ }^{1}$
}

1arc Epidemiology Unit, The University of Manchester, Manchester, UK ${ }^{2}$ Department of Rheumatology, Norfolk and Norwich University Hospital, Norwich, UK

\section{Correspondence to} Deborah P M Symmons, arc Epidemiology Unit, Manchester Academic Health Sciences Centre, University of Manchester, Stopford Building, Oxford Road, Manchester M13 9PT, UK:

deborah.symmons@ manchester.ac.uk

Accepted 31 January 2010

\begin{abstract}
Objectives Subjects with rheumatoid factor positive inflammatory polyarthritis (IP) are known to have increased mortality from cardiovascular disease (CVD). A study was undertaken to examine the risk and baseline predictors of admission with CVD in patients with recentonset IP.

Methods Subjects are recruited by the Norfolk Arthritis Register if they present to primary or secondary care with $\geq 2$ swollen joints lasting $\geq 4$ weeks. This analysis includes subjects recruited between 1995 and 1999. Baseline data on lifestyle, demographic characteristics, disease and treatment characteristics were collected. CVD admissions were identified through record linkage with the only acute care hospital in the study region. First-episode hospitalisation rates were compared with those of the general population. Poisson regression was used to calculate the relative risk (RR) of admission for patients with IP (overall and for each risk factor). Death certificates were obtained from the national death register.
\end{abstract}

Results 800 patients with recent-onset IP were followed for a median of 7.0 years. 64 CVD-related hospitalisations were observed (11.7 per 1000 person-years). Patients with IP were twice as likely ( $\mathrm{RR}=2.0 ; 95 \% \mathrm{Cl} 1.5$ to 2.5) to be hospitalised for CVD as the general population. Difficulty walking at baseline was a significant predictor of CVD admission and baseline non-steroidal antiinflammatory drug use was associated with a reduced risk of CVD admission.

Conclusions Patients with IP are at increased risk of CVD-related hospitalisation, within 7 years of symptom onset. Informing patients about lifestyle modification may reduce the risk of CVD.

\section{INTRODUCTION}

It is now well established that patients with rheumatoid arthritis (RA) have increased mortality from cardiovascular disease (CVD). ${ }^{1}{ }^{2}$ However, data from the Mayo clinic $^{3}$ and a previous UK study showed no increase in CVD admissions in patients with RA, despite an increase in CVD mortality. ${ }^{4}$ This raised the possibility that there is an increased case fatality among patients with RA admitted with CVD and/or more clinically silent disease. We sought to explore this issue further by comparing the rates of 'first' admission for CVD in a cohort of patients with early inflammatory polyarthritis (IP) (of which RA is a large subset) and in a matched sample of the general population. We also examined mortality from CVD over the same time period in this cohort.

\section{METHODS}

\section{Patients}

Patients recruited by the Norfolk Arthritis Register (NOAR), a primary care-based inception cohort based in Norwich, UK, with a symptom onset between 1995 and 1999 formed the study population. Recruitment methods for the NOAR cohort have been described in detail elsewhere. ${ }^{5}$ In brief, patients were referred to NOAR if they were aged at least 16 years and had consulted a physician within the study area for recent-onset synovitis affecting $\geq 2$ joints lasting $\geq 4$ weeks. Patients with a diagnosis other than RA, undifferentiated IP, psoriatic arthritis (PsA) or post-infective arthritis are excluded from NOAR, although the specific diagnoses of remaining patients are not recorded as they are within the classification of IP. Structured interviews and physical examinations were conducted by trained research nurses at baseline for all subjects. Baseline serum samples were later tested for rheumatoid factor $(R F)$, anti-cyclic citrullinated peptide antibody (ACPA) and C-reactive protein (CRP). Baseline Health Assessment Questionnaires (HAQs) ${ }^{6}$ were completed by all subjects. Patients were classified as 'physically inactive' if they reported any difficulty (some difficulty, much difficulty or unable to do) in response to the question: 'Are you able to walk outdoors on flat ground?' Patients were classified as past smokers, current or never smokers based on self-reported history of cigarette smoking. Finally, patients were classified as being exposed or not to any disease-modifying antirheumatic drugs (DMARDs, excluding steroids), methotrexate (MTX), steroids or non-steroidal anti-inflammatory drugs (NSAIDs, excluding lowdose aspirin) at baseline.

\section{Follow-up}

Patients were followed annually for 2 years after symptom onset. Subjects diagnosed at any time during follow-up with a condition (excluding RA, PsA or post-infective arthritis) that explained their symptoms were excluded from the analysis. The 1987 American College of Rheumatology (ACR) criteria for $\mathrm{RA}^{7}$ were applied cumulatively up to 2 years from symptom onset and subjects were categorised as having RA if they ever satisfied the criteria. The remaining subjects were categorised as having undifferentiated IP. All subjects were 'flagged' with the Office for National Statistics (ONS) who provided notification if the patient died together with a copy of the death certificate. 
The NOAR cohort data were linked to the electronic admissions system of the region's only acute hospital. This electronic admissions record system was established in January 1995. Record linkage enabled the identification of patients admitted to hospital between January 1995 and March 2004 including the date and reason for admission (coded using the International Classification of Diseases, 10th Revision (ICD10)). ${ }^{8}$ Patients admitted to hospital for any of the following conditions were included in the analysis: 'vascular disease' (any condition from Chapter I of ICD10); 'CVD' I00-I52.8; 'coronary heart disease' (CHD) I20-I25; or 'stroke' I60-I64. Length of follow-up was calculated from the date of onset of IP symptoms until the earliest of the date of first admission for a vascular event, death, emigration or 31 March 2004 (the end of the study period). For each grouping detailed above, hospitalisation rates per 1000 person years were calculated for the study population and stratified by gender. Mortality was studied for the same time period. Cause of death was taken from the death certificate.

Admissions data for the general Norfolk population were obtained from the regional public health observatory. These data were used to compute comparative age-, sex- and calendar year-specific hospitalisation rates for each category of vascular disease excluding heart failure (data on this outcome in the general population were unavailable). For both the NOAR cohort and the Norfolk population, only the first admission for vascular disease was considered throughout the follow-up period. Thus, each patient could only contribute one event in the current analysis.

ONS also provided death rates for the general Norfolk population from 1 January 1995 to 31 March 2004 for all-cause, 'vascular disease', CVD and CHD mortality.

\section{Statistical analysis}

Hospitalisation rates for the NOAR cohort were compared with those of the Norfolk population. These comparison rates were used as offset values in a Poisson regression to compute age, sex and calendar year relative risk (RR) (with their corresponding $95 \% \mathrm{CI}$ ) for groupings of vascular disease.

To compare the mortality risks of the NOAR cohort with those of the Norfolk population, standardised mortality ratios (SMRs) were estimated for all-cause, 'vascular disease' and CVD mortality by gender and within seropositive subgroups.

The association between lifestyle/demography, disease severity/activity and treatment factors and hospital admission for vascular disease was explored univariately within the cohort using Poisson regression. The lifestyle/demographic factors considered were: age at IP onset; gender (male vs female); smoking history at baseline (current vs never, past vs never); any difficulty walking at baseline (vs no difficulty). The following factors were considered as markers of disease severity/activity: cumulative RA status (ACR criteria, see above; yes vs no); baseline RF status (positive vs negative); baseline ACPA status (positive vs negative); baseline CRP concentration ( $\mathrm{mg} / \mathrm{l}$ ); and baseline $\mathrm{HAO}$ score. Finally, the following treatments were considered: baseline exposure to DMARDs, MTX, steroids or NSAIDs (yes vs no).

\section{RESULTS}

Between 1995 and the end of 1999, 800 patients were referred to the NOAR with a symptom onset after 1 January 1995 who did not receive a consultant diagnosis other than RA, PsA, postviral arthritis or undifferentiated IP during follow-up. Table 1 shows the characteristics of these patients. Linkage with the admissions database of the region's main hospital was possible for all 800 patients.

The patients were followed via the record linkage for a total of 5486 person-years from symptom onset, giving a median follow-up of 7.0 years (IOR 5.8-8.1). During this period 112 patients were hospitalised for a vascular condition. Hospitalisation rates for each grouping of vascular disease are shown in table 2. RR for first admission for each grouping of vascular disease compared with the expected regional hospitalisation rates are also shown in table 2 . The risk of hospitalisation for all vascular diseases combined, CVD and CHD was approximately doubled in patients with IP compared with the general population. There was no association between IP and hospitalisation for stroke.

During the same period 94 patients died, 53 of whom $(56.4 \%$ of all deaths) had a vascular condition recorded as the underlying cause of death. Table 3 shows the SMRs for all-cause, vascular disease and CVD mortality. All-cause mortality was slightly higher in the NOAR cohort than the local population but this was not statistically significantly. Mortality for vascular disease and CVD were also higher in the NOAR cohort but not significantly so. All-cause mortality was not increased in either the RF positive subgroup or the ACPA positive subgroup. CVD mortality was increased $53 \%$ in women who were positive for RF and was doubled in women who were positive for ACPA. However, these values were not statistically significant.

Table 4 shows the risk of admission for CVD associated with demographic, lifestyle and disease-related factors within the NOAR cohort. Increasing age and male gender were associated with an increased (unadjusted) risk of CVD admission. Patients reporting any difficulty walking as part of the $\mathrm{HAO}$ at baseline were nearly twice as likely to be hospitalised for CVD as those without any such difficulty. Interestingly, there was no significant association between baseline smoking status or CRP and risk of CVD, although this may be due to a lack of statistical power.

Table 1 Cohort characteristics at baseline

\begin{tabular}{lc}
\hline Characteristic & $\begin{array}{c}\text { n/N (\%), mean (SD) or } \\
\text { median (IOR)* }\end{array}$ \\
\hline Mean (SD) age at IP onset (years) & $55.4(16.6)$ \\
Median (IQR) symptom duration (months) & $6.4(3.6-12.0)$ \\
Gender: female, n/N (\%) & $536 / 800(67.0)$ \\
Smoking history & \\
$\quad$ Past smoker, n/N (\%) & $301 / 797(37.8)$ \\
Current smoker, n/N (\%) & $164 / 797(24.3)$ \\
$\quad$ Never smoker, n/N (\%) & $465 / 797(58.3)$ \\
Walking 5 m: any difficulty, n/N (\%) & $178 / 637(27.9)$ \\
Positive for rheumatoid factor, n/N (\%) & $213 / 717(29.7)$ \\
Positive for ACPA, n/N (\%) & $228 / 708(32.2)$ \\
Mean (SD) CRP concentration (mg/l) & $16.1(27.0)$ \\
Mean (SD) HAQ score & $0.84(0.74)$ \\
DMARD use, $\mathrm{n}(\%)$ & $253 / 800(31.6)$ \\
MTX use, $\mathrm{n}(\%)$ & $136 / 800(17.0)$ \\
Steroid use, $\mathrm{n} / \mathrm{N}(\%)$ & $134 / 800(16.8)$ \\
NSAID use, $\mathrm{n} / \mathrm{N}(\%)$ & $505 / 800(63.1)$ \\
Statin use, $\mathrm{n} / \mathrm{N}(\%)$ & $8 / 800(1.0)$ \\
Cumulative ACR criteria for RA after 2 years: & $387 / 800(48.4)$ \\
met criteria, $\mathrm{n} / \mathrm{N}(\%)$ & \\
\hline
\end{tabular}

*Denominator denotes number of patients for whom data were available. ACPA, anticyclic citrullinated peptide antibody; ACR, American College of Rheumatology; CRP, C-reactive protein; DMARD, disease-modifying antirheumatic drug; HAQ, Health Assessment Questionnaire; IP, inflammatory polyarthritis; MTX, methotrexate; NSAID, non-steroidal anti-inflammatory drug; RA, rheumatoid arthritis. 
Table 2 Admission rates for vascular disease and relative risk (RR) for first admission

\begin{tabular}{|c|c|c|c|c|c|c|c|}
\hline \multirow[b]{2}{*}{ Site (ICD10 codes) } & \multirow[b]{2}{*}{$\mathbf{N}$} & \multicolumn{3}{|c|}{ First admission rate per 1000 person-years $(95 \% \mathrm{CI})$} & \multirow[b]{2}{*}{ Observed } & \multirow[b]{2}{*}{ Expected } & \multirow[b]{2}{*}{$\mathbf{R R}(95 \% \mathrm{CI})$} \\
\hline & & Women & Men & Total & & & \\
\hline CVD (I00-153) & 64 & 8.1 (5.5 to 11.5$)$ & 19.2 (13.3 to 26.8 ) & 11.7 (9.0 to 14.9$)$ & 64 & 32.9 & $1.9(1.5$ to 2.5$)$ \\
\hline CHD (I20-126) & 32 & $3.0(1.5$ to 5.3$)$ & 11.8 (7.3 to 18.1$)$ & 5.8 (4.0 to 8.2 ) & 32 & 17.1 & 1.9 (1.6 to 2.3$)$ \\
\hline Stroke (160-164) & 9 & 1.6 (0.8 to 3.1$)$ & 1.7 (0.3 to 4.9$)$ & 1.6 (0.6 to 3.5$)$ & 9 & 8.4 & 1.1 (0.6 to 2.0$)$ \\
\hline $\begin{array}{l}\text { All vascular disease } \\
\text { (I00-I98X) }\end{array}$ & 112 & $15.4(11.6$ to 19.9$)$ & $31.0(23.3$ to 40.3$)$ & $20.4(16.8$ to 24.6$)$ & 112 & 54.8 & 2.0 (1.7 to 2.5$)$ \\
\hline
\end{tabular}

CHD, coronary heart disease; CVD, cardiovascular disease; ICD10, International Classification of Diseases, 10th Revision.

Table 3 SMRs for all-cause, vascular disease and CVD mortality by gender, overall and for seropositive subgroups

\begin{tabular}{|c|c|c|c|}
\hline & \multicolumn{3}{|c|}{ Main cause of death } \\
\hline & All-cause & Vascular disease & CVD \\
\hline & SMR (95\% Cl) & SMR $(95 \% \mathrm{CI})$ & SMR (95\% CI) \\
\hline \multicolumn{4}{|c|}{ Whole cohort } \\
\hline Men & $1.02(0.73$ to 1.38$)$ & $1.06(0.63$ to 1.68$)$ & 0.95 (0.47 to 1.70$)$ \\
\hline Women & $1.16(0.87$ to 1.52$)$ & $1.21(0.76$ to 1.83$)$ & 1.52 (0.87 to 2.48 ) \\
\hline Total & $1.10(0.88$ to 1.34$)$ & $1.14(0.81$ to 1.56$)$ & 1.22 (0.81 to 1.78 ) \\
\hline \multicolumn{4}{|c|}{ RF-positive subgroup } \\
\hline Men & $1.12(0.61$ to 1.87$)$ & $0.93(0.30$ to 2.16$)$ & 1.07 (0.29 to 2.74$)$ \\
\hline Women & 1.20 (0.66 to 2.02$)$ & $1.11(0.36$ to 2.59$)$ & 1.53 (0.42 to 3.91$)$ \\
\hline Total & 1.16 (0.69 to 1.23$)$ & $1.01(0.48$ to 1.86$)$ & 1.26 (0.54 to 2.48$)$ \\
\hline \multicolumn{4}{|c|}{ ACPA-positive subgroup } \\
\hline Men & $1.18(0.65$ to 1.98$)$ & 0.79 (0.21 to 2.02 ) & 0.85 (0.18 to 2.49 ) \\
\hline Women & $1.25(0.68$ to 2.10$)$ & 1.42 (0.52 to 3.08$)$ & 2.01 (0.65 to 4.68 ) \\
\hline Total & $1.22(0.81$ to 1.76$)$ & 1.07 (0.51 to 1.97$)$ & 1.33 (0.57 to 2.62 ) \\
\hline
\end{tabular}

ACPA, anticyclic citrullinated peptide antibody; CVD, cardiovascular disease; RF, rheumatoid factor; SMR, standardised mortality ratio

Finally, the risk of admission for CVD within the NOAR cohort was explored by patient baseline treatment status (table 5). Exposure to any DMARD, MTX or steroids was not associated with a significantly increased (adjusted) risk. However, patients who were taking NSAIDs at baseline were half as likely to be admitted for CVD as patients who were not using these agents.

\section{DISCUSSION}

Results from the present study show that, in Norfolk, patients with a recent onset of IP were twice as likely to be hospitalised for a vascular condition as the general population (adjusted for age and gender). A similar doubling of risk was observed for $\mathrm{CVD}$ and $\mathrm{CHD}$ (as defined in this study), although no increase in hospital admission for stroke was observed. Difficulty in walking was the only factor associated with a significant increase in CVD admission risk in patients with IP. We also found a modest increase in CVD mortality which was more marked in women. This increase in mortality was less marked than that observed in patients recruited by NOAR with a symptom onset between 1990 and 1994 after a similar length of follow-up. ${ }^{19}$ As in our previous studies, the increased risk of CVD mortality was mainly seen in RF-positive and ACPA-positive women, although the results did not reach statistical significance in the present study.

Interestingly, the findings of this study are in contrast to those of a similar study based in Stockport, UK. ${ }^{4}$ In that study, Goodson et al studied the risk of death from and hospital admission for 'all vascular disease' in a population of patients with RA, as identified through rheumatology clinics. Patients in their cohort were 1.4 times (men) and 1.9 times (women) more likely to die from vascular disease than the general population.
Table 4 Demographic/lifestyle and disease-related predictors of CVD admission

\begin{tabular}{|c|c|c|c|c|c|}
\hline Factor & $\begin{array}{l}\text { Number with CVD } \\
\text { admission } \\
\text { (\% of group) }\end{array}$ & $\begin{array}{l}\text { Unadjusted } \\
\text { RR }\end{array}$ & $95 \% \mathrm{Cl}$ & $\begin{array}{l}\text { Adjusted* } \\
\text { RR }\end{array}$ & $95 \% \mathrm{Cl}$ \\
\hline $\begin{array}{l}\text { Age at symptom } \\
\text { onset (per decade) }\end{array}$ & 64 & 1.8 & 1.5 to 2.2 & - & - \\
\hline \multicolumn{6}{|l|}{ Gender } \\
\hline Female & $30(5.6)$ & 1 & Referent & - & - \\
\hline Male & $34(12.9)$ & 2.4 & 1.5 to 4.0 & - & - \\
\hline \multicolumn{6}{|l|}{ Smoker } \\
\hline Never & $20(4.3)$ & 1 & Referent & 1 & Referent \\
\hline Past & $25(8.3)$ & 1.3 & 0.7 to 2.3 & 0.8 & 0.4 to 1.4 \\
\hline Current & $19(11.6)$ & 1.6 & 0.8 to 2.9 & 1.5 & 0.8 to 2.8 \\
\hline \multicolumn{6}{|l|}{ RA by 2 years } \\
\hline No & $26(6.3)$ & 1 & Referent & 1 & Referent \\
\hline Yes & $38(9.8)$ & 1.6 & 0.96 to 2.6 & 1.2 & 0.7 to 1.9 \\
\hline \multicolumn{6}{|l|}{ Baseline RF } \\
\hline -ve & $40(7.9)$ & 1 & Referent & 1 & Referent \\
\hline +ve & $18(8.5)$ & 0.92 & 0.5 to 1.6 & 0.8 & 0.5 to 1.5 \\
\hline \multicolumn{6}{|l|}{ Baseline ACPA } \\
\hline- ve & $36(7.5)$ & 1 & Referent & 1 & Referent \\
\hline$+\mathrm{ve}$ & $21(9.2)$ & 1.2 & 0.7 to 2.1 & 1.1 & 0.7 to 2.0 \\
\hline $\begin{array}{l}\text { CRP concentration } \\
\text { (mg/l) }\end{array}$ & 57 & 1.0 & 0.99 to 1.0 & 0.99 & $\begin{array}{l}0.99 \text { to } \\
1.0\end{array}$ \\
\hline Baseline HAO & 64 & 1.1 & 0.8 to 1.5 & 1.1 & 0.8 to 1.6 \\
\hline \multicolumn{6}{|l|}{ Walk } \\
\hline No difficulty & $31(6.8)$ & 1 & Referent & 1 & Referent \\
\hline Any difficulty & $26(14.6)$ & 2.3 & 1.4 to 4.0 & 2.0 & 1.2 to 3.5 \\
\hline
\end{tabular}

${ }^{*}$ Adjusted for age, gender and calendar year.

ACPA, anticyclic citrullinated peptide antibody; CRP, C-reactive protein; CVD, cardiovascular disease; $\mathrm{HAQ}$, Health Assessment Questionnaires; RA, rheumatoid arthritis; RF, rheumatoid factor; RR, relative risk.

However, the study population was no more likely to be hospitalised for vascular disease than the general population. This contrast in results could be due to differences in the patients studied (early RA vs early IP). It is possible that patients in our 'milder' IP cohort are more likely to have 'milder' CVD eventsthat is, resulting in hospitalisation rather than death-than the early RA cohort. Also, the Stockport study considered all hospital admissions per patient whereas our analysis compared the rate of first (post-IP onset) hospitalisation only. On the basis of their results, Goodson et al ${ }^{4}$ hypothesised that patients with RA have an increased post-CVD event case fatality compared with the general population.

Certain results from this study were somewhat unexpected. First, it is widely recognised that the risk of CVD in the general population is increased in subjects with a history of smoking 1011 as well as a raised high sensitivity CRP concentration. ${ }^{11-13}$ Our study failed to detect a significantly increased risk of CVD with either of these factors. A lack of association between smoking and cardiovascular mortality has previously been reported in NOAR ${ }^{1}$ and other RA cohorts. ${ }^{14}$ 
Table 5 Baseline treatment predictors of CVD admission

\begin{tabular}{|c|c|c|c|c|c|}
\hline Factor & $\begin{array}{l}\text { Number of cases } \\
\text { of CVD (\%) }\end{array}$ & $\begin{array}{l}\text { Unadjusted } \\
\text { RR }\end{array}$ & $95 \% \mathrm{Cl}$ & $\begin{array}{l}\text { Adjusted* } \\
\text { RR }\end{array}$ & $95 \% \mathrm{CI}$ \\
\hline \multicolumn{6}{|c|}{ DMARDs } \\
\hline No & $42(7.7)$ & 1 & Referent & 1 & Referent \\
\hline Yes & $22(8.7)$ & 1.1 & 0.7 to 1.9 & 1.2 & 0.7 to 2.0 \\
\hline \multicolumn{6}{|l|}{ MTX } \\
\hline No & $50(7.5)$ & 1 & Referent & 1 & Referent \\
\hline Yes & $14(10.3)$ & 1.4 & 0.8 to 2.6 & 1.2 & 0.7 to 2.2 \\
\hline \multicolumn{6}{|c|}{ Steroids } \\
\hline No & $45(6.8)$ & 1 & Referent & 1 & Referent \\
\hline Yes & $19(14.2)$ & 2.4 & 1.4 to 4.2 & 1.2 & 0.7 to 2.0 \\
\hline \multicolumn{6}{|l|}{ NSAID } \\
\hline No & $36(12.2)$ & 1 & Referent & 1 & Referent \\
\hline Yes & $28(5.5)$ & 0.5 & 0.3 to 0.8 & 0.6 & 0.3 to 0.9 \\
\hline
\end{tabular}

${ }^{*}$ Adjusted for age, gender and calendar year.

CVD, cardiovascular disease; DMARDs, disease-modifying antirheumatic drugs; MTX, methotrexate; NSAID, non-steroidal anti-inflammatory drug; RR, relative risk.

The degree of increase in CVD SMR in RF- and ACPA-positive patients has fallen based on the findings in NOAR patients recruited between 1990 and $1994 .{ }^{19}$ In the 1990-4 cohort the SMR in RF-positive women in NOAR was 2.02 compared with 1.53 in the present study. There are a number of possible explanations for this. The natural history of RF-positive IP may be changing and the disease may be becoming milder. In addition, disease-modifying therapy is becoming more aggressive and is started earlier. Thus, any excess in CVD mortality may not be seen until later in the disease course. This is likely if the cumulative burden of inflammation is a significant predictor of excess cardiovascular mortality in patients with RF-positive IP. It is also possible that the management of CVD has improved and so patients are surviving longer after their first manifestation of CVD. Another reason for the differences in the findings between this and earlier studies may be the switch from ICD versions 9-10 (used from 1994) to classify the cause of death. The diseases included in the 'circulatory disease' chapter in version 9 do not directly map to the equivalent chapter in version 10. Further follow-up of this cohort is needed to see if the SMR due to CVD in RF-positive patients changes with time.

This study detected a reduced risk of CVD in patients in NOAR who were exposed to NSAIDs at baseline compared with those who were not. This finding is unexpected given the recent publicity with regard to a potential link between NSAID exposure and CVD mortality. ${ }^{15}$ However, we have recently reported that NSAID use assessed cumulatively in patients from NOAR is not associated with increased cardiovascular mortality. ${ }^{16}$ In our cohort, this finding may represent a 'healthy user' effect in terms of which patients are prescribed NSAIDs.

This is the first study to explore the risk of admission for vascular disease in a primary care-based population of patients with recent-onset IP. The study is unaffected by the potential recruitment bias often associated with studies focusing on patients with RA recruited solely through hospitals or clinics. A particular strength of this study was the focus on patients with IP of very recent onset and the possibility of record linkage from the date of symptom onset, thus minimising left censorship.

However, there are some methodological issues that require consideration. Record linkage could only be achieved from 1995 onwards. This coincided with a 5-year recruitment period in NOAR (1995-9) in which most subjects were only followed for 2 years from symptom onset. We are therefore unable to analyse, for example, cumulative disease activity or drug exposure as a predictor of subsequent admission. The study cohort includes patients with undifferentiated IP as well as patients who could be classified as having RA according to the ACR criteria. ${ }^{7}$ The increased risk observed in this study may therefore underestimate the level of risk in patients with RA. The ACR criteria are known to be relatively unstable in early arthritis, ${ }^{17-19}$ and thus we feel the focus on IP is justifiable. In this study population, slightly less than half of the patients met the ACR criteria applied cumulatively by 2 years from symptom onset. Importantly, the risk of hospital admission for CVD in the RA subgroup was no different from that of the complete cohort ( $\mathrm{RR}=1.8,95 \%$ CI 1.2 to 2.7$)$ and the results for the predictive analyses were essentially identical. Also, we have previously shown that RA status is a poor predictor of CVD mortality. ${ }^{1}$ We did not have access to individual patient level data for the background Norfolk population which may have been insightful, such as traditional CVD risk factors or drug treatment history.

Difficulty in walking may be viewed as a lifestyle factor for the risk of CVD as these patients are less mobile than those with no difficulty and people who take more exercise are probably at a lower risk of a CVD event. It may also be viewed as a disease-related factor because patients with the most severe disease will have the greatest physical disability preventing them from walking. An approach that encompasses both good disease management and improvement in lifestyle may reduce the risk of CVD.

Acknowledgements The authors thank the rheumatologists and general practitioners within the catchment region for their continued support of the Norfolk Arthritis Register (NOAR). They also acknowledge the contribution of the NOAR metrologists in the collection of the clinical data and database manager Stephanie Manning, and thank the Norfolk Public Health Observatory for providing the data regarding hospital admissions.

Funding This work was funded by the Arthritis Research Campaign, UK (grant reference 17552).

\section{Competing interests None.}

Ethics approval This study was conducted with the approval of the Norfolk and Norwich local research ethics committee.

Provenance and peer review Not commissioned; externally peer reviewed.

\section{REFERENCES}

1. Goodson NJ, Wiles NJ, Lunt M, et al. Mortality in early inflammatory polyarthritis: cardiovascular mortality is increased in seropositive patients. Arthritis Rheum 2002;46:2010-19.

2. Aviña-Zubieta JA, Choi HK, Sadatsafavi M, et al. Risk of cardiovascular mortality in patients with rheumatoid arthritis: a meta-analysis of observational studies. Arthritis Rheum 2008;59:1690-7.

3. Gabriel SE, Crowson CS, O'Fallon WM. Comorbidity in arthritis. J Rheumatol 1999;26:2475-9.

4. Goodson N, Marks J, Lunt M, et al. Cardiovascular admissions and mortality in an inception cohort of patients with rheumatoid arthritis with onset in the 1980s and 1990s. Ann Rheum Dis 2005;64:1595-601.

5. Symmons DP, Silman AJ. The Norfolk Arthritis Register (NOAR). Clin Exp Rheumatol 2003;21 (5 Suppl 31):S94-9.

6. Fries JF, Spitz P, Kraines RG, et al. Measurement of patient outcome in arthritis. Arthritis Rheum 1980;23:137-45

7. Arnett FC, Edworthy SM, Bloch DA, et al. The American Rheumatism Association 1987 revised criteria for the classification of rheumatoid arthritis. Arthritis Rheum 1988;31:315-24.

8. World Health Organization. International classification of diseases, 10th revision. Geneva: World Health Organization, 1994.

9. Naz SM, Farragher TM, Bunn DK, et al. The influence of age at symptom onset and length of followup on mortality in patients with recent-onset inflammatory polyarthritis. Arthritis Rheum 2008;58:985-9.

10. Wolfe F, Michaud K. Heart failure in rheumatoid arthritis: rates, predictors, and the effect of anti-tumor necrosis factor therapy. Am J Med 2004;116:305-11. 
11. Kremers HM, Crowson CS, Therneau TM, et al. High ten-year risk of cardiovascular disease in newly diagnosed rheumatoid arthritis patients: a population-based cohort study. Arthritis Rheum 2008;58:2268-74.

12. Berton G, Cordiano R, Palmieri R, et al. C-reactive protein in acute myocardial infarction: association with heart failure. Am Heart J 2003;145:1094-101.

13. Bursi F, Weston SA, Killian JM, et al. C-reactive protein and heart failure after myocardial infarction in the community. Am J Med 2007;120:616-22.

14. Solomon DH, Curhan GC, Rimm EB, et al. Cardiovascular risk factors in women with and without rheumatoid arthritis. Arthritis Rheum 2004;50:3444-9.

15. Johnsen SP, Larsson H, Tarone RE, et al. Risk of hospitalization for myocardia infarction among users of rofecoxib, celecoxib, and other NSAIDs: a population-based case-control study. Arch Intern Med 2005:165:978-84.
16. Goodson NJ, Brookhart AM, Symmons DP, et al. Non-steroidal anti-inflammatory drug use does not appear to be associated with increased cardiovascular mortality in patients with inflammatory polyarthritis: results from a primary care based inception cohort of patients. Ann Rheum Dis 2009;68:367-72.

17. Wiles N, Symmons DP, Harrison B, et al. Estimating the incidence of rheumatoid arthritis: trying to hit a moving target? Arthritis Rheum 1999;42:1339-46.

18. MacGregor AJ, Bamber S, Silman AJ. A comparison of the performance of different methods of disease classification for rheumatoid arthritis. Results of an analysis from a nationwide twin study. J Rheumatol 1994;21:1420-6.

19. Harrison BJ, Symmons DP, Barrett EM, et al. The performance of the 1987 ARA classification criteria for rheumatoid arthritis in a population based cohort of patients with early inflammatory polyarthritis. J Rheumatol 1998;25:2324-30. 